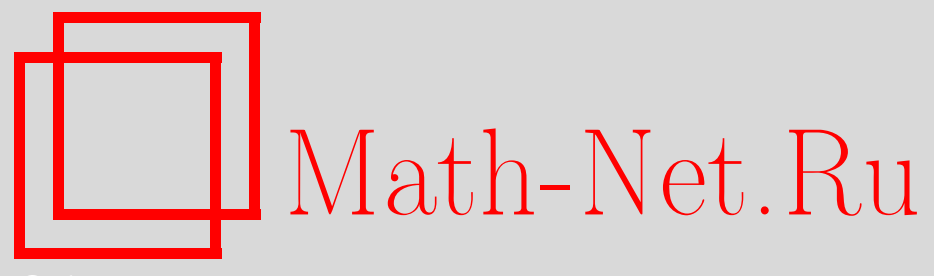

Д. Греб, Х. Лен, З. Ролленске, Лагранжевы слоения на четырехмерных гиперкэлеровых многообразиях, Изв. РАН. Сер. матем., 2014, том 78, выпуск 1, 25-36

DOI: https://doi.org/10.4213/im7973

Использование Общероссийского математического портала Math-Net.Ru подразумевает, что вы прочитали и согласны с пользовательским соглашением http://www.mathnet.ru/rus/agreement

Параметры загрузки:

IP : 3.89 .185 .249

26 апреля 2023 г., 14:55:52

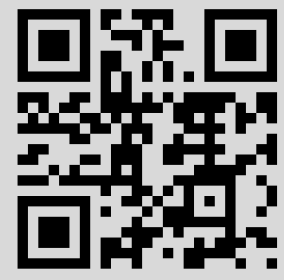


УДК 514.154

\author{
Д. Греб, Х. Лен, З. Ролленске
}

\title{
Лагранжевы слоения на четырехмерных гиперкэлеровых многообразиях
}

\begin{abstract}
В качестве ответа на усиленный вариант вопроса, поставленного А. Бовиллем, дается короткое геометрическое доказательство того, что всякое четырехмерное гиперкэлерово многообразие, содержащее лагранжев подтор $L$, допускает голоморфное лагранжево слоение со слоем $L$.
\end{abstract}

Библиография: 12 наименований.

Ключевые слова: гиперкэлерово многообразие, лагранжево слоение.

DOI: $10.4213 / \operatorname{im} 7973$

\section{§ 1. Введение}

Пусть $X$ - гиперкэлерово многообразие, т. е. компактное односвязное кэлерово многообразие, для которого векторное пространство $H^{0}\left(X, \Omega_{X}^{2}\right)$ порождается некоторой голоморной симплектической формой $\sigma$. Из работ Д. Мацушиты хорошо известно, что любое нетривиальное голоморфное отображение из $X$ в комплексное пространство меньшей размерности является лагранжевым слоением (см. §2). Более того, частный случай так называемой гиперкэлеровой SYZ-гипотезы гласит, что всякое гиперкэлерово многообразие можно продеформировать в гиперкэлерово многообразие, допускающее лагранжево слоение.

Поэтому важной задачей является нахождение геометрических условий для гиперкэлерова многообразия, которые гарантировали бы существование на нем лагранжева слоения. Следующий вопрос был поставлен А. Бовиллем [1, п. 1.6].

Вопрос А. Пусть $X$ - гиперкэлерово многообразие, а $L$ - лагранжев тор в $X$. Является ли тогда $L$ слоем некоторого (мероморфного) лагранжева слоения $f: X \rightarrow B$ ?

В нашей предыдущей статье [2] был дан положительный ответ на вопрос А в случае непроективного $X$. Более того, для всякого гиперкэлерова многообразия, допускающего почти голоморфное лагранжево слоение, было найдено бирационально эквивалентное ему гиперкэлерово многообразие, на котором это лагранжево слоение становится голоморфным.

Развиваемый в настоящей работе подход к решению вопроса Бовилля в проективном случае основан на детальном изучении теории деформаций тора $L$ в многообразии $X$. Для этого рассмотрим компоненту $\mathfrak{B}$ пространства Барлета, содержащую $[L]$, вместе с универсальным семейством и вычисляющим отображением со значениями в $X$ :

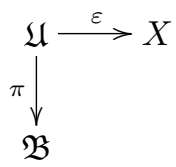

(C) Д. Греь, Х. ЛЕн, З. РолЛЕнске, 2014 
В [2, лемма 3.1] было показано, что отображение $\varepsilon$ сюръективно и его слои в общем положении конечны, а также что $X$ допускает почти голоморфное лагранжево слоение тогда и только тогда, когда $\operatorname{deg}(\varepsilon)=1$.

Если степень отображения $\varepsilon$ строго больше единицы, то некоторые деформации тора $L$ пересекают $L$ неожиданным образом. Для изучения этой ситуации введем понятие $L$-редукиии: для каждого проективного гиперкэлерова многообразия, содержащего лагранжев тор, найдутся проективное многообразие $\mathfrak{T}$ и рациональное отображение $\varphi_{L}: X \rightarrow \mathfrak{T}$, единственное с точностью до бирациональной эквивалентности, слой которого, проходящий через общую точку $x$, равен связной компоненте пересечения всех деформаций тора $L$, проходящих через $x$. Многообразие $X$ назовем $L$-отделимым, если отображение $\varphi_{L}$ бирационально. Мы докажем следующий результат (теорема 3.5): nycmъ $X$ - npoективное гиперкэлерово многообразие, а $L \subset X$ - лагранжев подтор; тогда $X$ допускает почти голоморфное слоение с сильным слоем $L$ в том и только в том случае, когда $X$ не является L-отделимым.

Для четырехмерных гиперкэлеровых многообразий $X$ можно исключить случай $L$-отделимости $X$ с помощью симплектической линейной алгебры. Более того, детальное знание бирациональной геометрии четырехмерных гиперкэлеровых многообразий дает положительный ответ на самый сильный вариант вопроса Бовилля (теорема 5.1): пусть $X$ - четырехмерное гиперкэлерово многообразие, содержащее лагранжев тор L; тогда $X$ допускает голоморфное лагранжево слоение со слоем $L$.

На конференции "Геометрические структуры на комплексных многообразиях" в Москве Е. Америк обратила наше внимание на следующий результат, независимо полученный ею на основе одного замечания из [3]: в размерности четыре всякое проективное гиперкэлерово многообразие, содержащее лагранжев подтор $L$, допускает почти голоморфное лагранжево слоение со слоем $L$ [4].

Уже после того, как наша статья была написана, появился препринт Хуанга и Вайсса, где для проективного случая дан ответ на ослабленный вариант вопроса Бовилля: построено почти голоморфное лагранжево слоение на любом проективном $2 n$-мерном гиперкэлеровом многообразии, содержащем лагранжев тор (см. [5]). Их рассуждение состоит из двух частей: геометрической и относящейся к абстрактной теории групп. В отличие от этого наш ответ (теорема 5.1) на силъный вариант вопроса Бовилля является чисто геометрическим, опирается на глобальные рассуждения в дополнение к локальным соображениям теории деформаций, а вместо теоретико-групповой части использует симплектическую линейную алгебру.

Авторы признательны Д. Хюбрехтсу за интерес к работе и ряд стимулирующих обсуждений. Благодарим Е. Америк, сообщившую нам наблюдение, содержащееся в лемме 5.3, которое сильно упростило наше предыдущее рассуждение. Второй автор благодарен Л. Манивелю за стимулирующие обсуждения и, в частности, за указание на замечание 5.4. Третий автор благодарит М. Вербицкого за приглашение в Москву.

Для успеха нашего сотрудничества была неоценимой поддержка Немецкого исследовательского общества (DFG) по проекту SFB/TR 45, исследовательская группа 790, а также поддержка работы третьего автора по проекту Э. Нётер. Первый автор благодарен фонду Бадена-Вюртемберга за поддержку по линии "Eliteprogramm für Postdoktorandinnen und Postdoktoranden". Второй ав- 
тор благодарит за поддержку Национальный совет по научным исследованиям (CNRS) и институт Фурье.

\section{§ 2. Предварительные сведения и обозначения}

\section{1. Лагранжевы слоения. Дадим следующее}

ОПРЕДЕЛЕНИЕ 2.1. Пусть $X$ - гиперкЭлерово многообразие. Лагранжевым слоением на $X$ называется голоморфное сюръективное отображение $f: X \rightarrow B$ со связными слоями на нормальное комплексное пространство $B$ такое, что каждая неприводимая компонента редукции каждого слоя $f$ является лагранжевым подмногообразием в $X$.

Из фундаментальных результатов Мацушиты вытекает, что всякое слоение на гиперкэлеровом многообразии автоматически лагранжево.

Теорема 2.2 [6]-[9]. Пусть $X$ - гиперкэлерово многообразие размерности $2 n$, a $f: X \rightarrow B$ - морфизм со связными слоями в нормальное комплексное пространство $B$, где $0<\operatorname{dim} B<\operatorname{dim} X$. Тогда $f$ является лагранжевым слоением. В частности, $f$ равноразмерностно ${ }^{1}, u \operatorname{dim} B=n$. При этом каждый гладкий слой отображения $f$ является комплексным тором.

2.2. Мероморфные отображения. Пусть $X$ - нормальное комплексное пространство, $Y$ - компактное комплексное пространство, а $f: X \rightarrow Y$ - мероморфное отображение. Рассмотрим разрешение неопределенностей

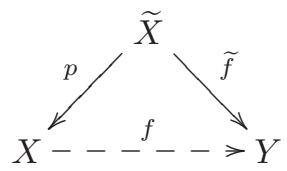

отображения $f$. Определим слой $F_{y}$ отображения $f$ над точкой $y \in Y$ по формуле $F_{y}:=p\left(\widetilde{f}^{-1}(y)\right)$. Это определение не зависит от выбора разрешения неопределенностей.

Напомним, что мероморфное отображение $f: X \rightarrow Y$ указанного выше вида называется почти голоморфным, если существует открытое по Зарисскому подмножество $U \subset Y$ такое, что сужение $\left.f\right|_{f^{-1}(U)}: f^{-1}(U) \rightarrow U$ есть собственное голоморфное отображение. Слой отображения $\left.f\right|_{f^{-1}(U)}$ называется сильнъм слоем почти голоморфного отображения $f$.

Пусть $X$ - нормальное алгебраическое многообразие, $B$ - полное алгебраическое многообразие, а $f: X \rightarrow B-$ почти голоморфное рациональное отображение. Для любого дивизора $A$ на $B$ можно определить его прообраз при $f$ либо геометрически, как замыкание прообраза $A$ на множестве точек голоморфности $f$, либо на уровне локально свободных пучков по формуле $f^{*} \mathscr{O}_{B}(A):=\left(p_{*} \tilde{f}^{*} \mathscr{O}_{B}(A)\right)^{\vee \vee}$, где $p: \widetilde{X} \rightarrow X-$ любое разрешение неопределенностей, как на диаграмме (2.1).

2.3. Деформации лагранжевых подторов. Отправной точкой нашего подхода к решению вопроса Бовилля является теория деформаций лагранжева

\footnotetext{
${ }^{1}$ То есть все слои отображения $f$ имеют одинаковые размерности.
} 
подтора $L$ в гиперкэлеровом многообразии $X$. Напомним кратко нужные нам результаты из $[2, \S 2,3]$.

Пространство Барлета $\mathfrak{B}(X)$ многообразия $X$ (или схема Чжоу в проективном случае) параметризует компактные циклы на $X$. Оказывается (см. ниже п. (i) леммы 2.3), что существует единственная неприводимая компонента $\mathfrak{B}$ пространства $\mathfrak{B}(X)$, содержащая точку $[L]$. Обозначая через $\mathfrak{U}$ график универсального семейства над $\mathfrak{B}$, а через $\Delta$ - дискриминантное множество компоненты $\mathfrak{B}$ (т. е. множество точек, параметризующих сингулярные элементы семейства $\mathfrak{B})$, получаем следующую диаграмму:

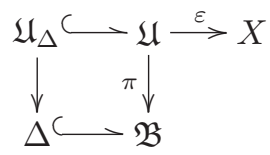

Подробный анализ отображений, входящих в диаграмму (2.2), показывает, что малая этальная или аналитическая окрестность подмногообразия $L$ в $X$ расслаивается над окрестностью точки $[L]$ в $\mathfrak{B}$. Точнее, имеем следующий результат.

Лемма 2.3 [2, лемма 3.1]. Пусть $X$ - гиперкэлерово многообразие размерности $2 n$, a $L$-лагранжев подтор в $X$. Тогда справедливы следующие утверждения:

(i) пространство Барлета $\mathfrak{B}(X)$ гладко и имеет размерность $n$ вблизи $[L]$; в частности, $[L]$ содержится в единственной неприводимой компоненте $\mathfrak{B}$ пространства $\mathfrak{B}(X)$, а $\mathfrak{U}$ является гладким и имеет размерность 2 в вблизи множества $\pi^{-1}([L])$;

(ii) морфизм $\varepsilon$ конечен и этален вдоль гладких слоев отображсния $\pi$; в частности, достаточно малые деформачии $L$ не пересекаются $c L$ и не существует семейств положительной размерности из гладких слоев, проходящих через точку $x \in X$ общего положения;

(iii) если $\left[L^{\prime}\right] \in \mathfrak{B}$ для гладкого $L^{\prime}$, то $L^{\prime}$ является лагранжевым подтором в $X$.

ЗАмечАниЕ 2.4. Приведем два простых, но полезных следствия леммы 2.3.

(i) Множество $X_{\Delta}:=\varepsilon\left(\mathfrak{U}_{\Delta}\right)$ состоит из тех точек $x \in X$, для которых существует сингулярная деформация тора $L$, проходящая через $x$. По соображениям размерности множество $X_{\Delta}$ является собственным подмножеством в $X$, а по лемме 2.3 , (ii) отображение $\varepsilon$ конечно и этально на прообразе множества $X \backslash X_{\Delta}$.

(ii) Из утверждения (ii) леммы 2.3 вытекает, в частности, что для любых двух точек $[L],[M] \in \mathfrak{B}$ их произведение-пересечение $[L] .[M]$ как циклов в $X$ равно нулю. Поэтому члены семейства $\mathfrak{B}$ не могут пересекаться по конечному числу точек.

\section{4. Почти голоморфные лагранжевы слоения и пространства Бар-} лета. Следующий результат связывает обсуждавшуюся выше теорию деформаций $L$ в $X$ с нашим вопросом о глобально определенных почти голоморфных лагранжевых слоениях.

Лемма 2.5 [2, лемма 3.2]. Пусть $X$ - гиперкэлерово многообразие, содержащее лагранжев подтор $L$. Тогда $X$ допускает почти голоморфное лагранжево слоение с сильным слоем L в том и только в том случае, если вычисляющее отображение є из диаграммы (2.2) бимероморфно. 
Если отображение $\varepsilon$ бирационально, то $\pi \circ \varepsilon^{-1}-$ искомое почти голоморфное слоение (с точностью до нормализации пространства $\mathfrak{B})$. Обратное доказывается с помощью пространства Барлета разрешения неопределенностей.

\section{§ 3. $L$-редукция и $L$-отделимые многообразия}

Пусть $X$ - проективное гиперкэлерово многообразие, содержащее лагранжев подтор L. В этом параграфе начинается наш анализ отображений, входящих в диаграмму (2.2). Напомним, что согласно лемме 2.5 для положительного ответа на вопрос Бовилля надо установить бирациональность отображения $\varepsilon$.

3.1. L-редукция. Здесь строится мероморфное отображение, ассоциированное с накрывающим семейством $\left\{L_{t}\right\}_{t \in \mathfrak{B}}$. В общей точке это отображение является факторизацией по мероморфному отношению эквивалентности, заданному семейством $\left\{L_{t}\right\}$, т. е. отождествляет те точки из $X$, которые не разделяются элементами из $\left\{L_{t}\right\}$.

3.1.1. Построение $L$-редукции. Пользуясь обозначениями из диаграммы $(2.2)$, положим $\mathfrak{U}_{\text {reg }}:=\varepsilon^{-1}\left(X \backslash X_{\Delta}\right)$. Напомним, что по замечанию 2.4 отображение

$$
\left.\varepsilon\right|_{\mathfrak{U}_{\mathrm{reg}}}: \mathfrak{U}_{\mathrm{reg}} \rightarrow X \backslash X_{\Delta}
$$

конечно и этально. Обозначим его степень через $d$.

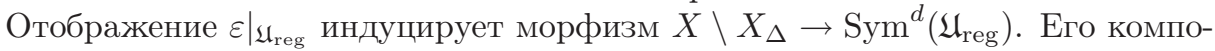
зиция с естественным морфизмом $\operatorname{Sym}^{d}\left(\mathfrak{U}_{\mathrm{reg}}\right) \rightarrow \operatorname{Sym}^{d}(\mathfrak{B})$, индуцированным отображением $\pi: \mathfrak{U} \rightarrow \mathfrak{B}$, дает морфизм $X \backslash X_{\Delta} \rightarrow \operatorname{Sym}^{d}(\mathfrak{B})$. Последний естественно продолжается до рационального отображения $\psi: X \rightarrow \operatorname{Sym}^{d}(\mathfrak{B})$. Пусть

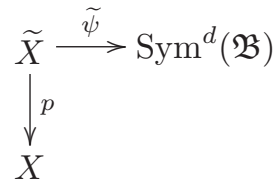

есть разрешение неопределенностей отображения $\psi$ с неособым $\widetilde{X}$. Тогда факторизация Штейна для $\widetilde{\psi}$ дает следующую диаграмму:

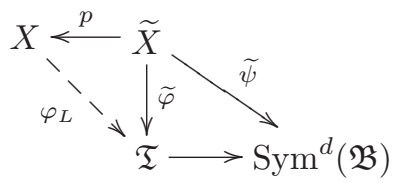

Здесь $\varphi_{L}=\widetilde{\varphi} \circ p^{-1}: X \rightarrow \mathfrak{T}-$ рациональное отображение, индуцированное отображением $\widetilde{\varphi}$. Заметим, что отображение $\varphi_{L}: X \rightarrow \mathfrak{T}$ единственно с точностью до бирациональной эквивалентности и, тем самым, канонически ассоциировано с парой $(X, L)$. Назовем его $L$-редукиией многообразия $X$.

ЗАмечание 3.1. Для каждой точки $x \in X \backslash X_{\Delta}$ в семействе $\left\{L_{t}\right\}_{t \in \mathfrak{B}}$ имеются $d$ различных гладких торов $L_{1}, \ldots, L_{d}$, содержащих $x$. По построению отображение $\varphi_{L}$ определено в точке $x$ и переводит ее в класс набора $\left(\left[L_{1}\right], \ldots,\left[L_{d}\right]\right)$ B $\operatorname{Sym}^{d}(\mathfrak{B})$. 
3.1.2. Простейшие свойства $L$-редукции. Следующее теоретико-множественное утверждение немедленно вытекает из конструкции отображения $\varphi_{L}$.

Лемма 3.2. Слой отображения $\varphi_{L}$, проходящий через точку $x \in X \backslash X_{\Delta}$, совпадает со связной компонентой множества

$$
\bigcap_{[M] \in \mathfrak{B}, x \in M} M,
$$

содержащей $x$.

ДокАЗАтЕльство. Если $x \in X \backslash X_{\Delta}$, то отображение $\varepsilon$ этально в каждой точке прообраза $\varepsilon^{-1}(x)$. Поэтому образ $\pi\left(\varepsilon^{-1}(x)\right)=\left\{\left[L_{1}\right], \ldots,\left[L_{d}\right]\right\}$ состоит из тех точек пространства $\mathfrak{B}$, которые параметризуют $d$ различных подторов в $X$, проходящих через $x$. В частности, мероморфное отображение $\psi: X--\rightarrow$ $\operatorname{Sym}^{d}(\mathfrak{B})$ определено в точке $x$, и его слой есть

$$
\psi^{-1}(\psi(x))=\bigcap_{i} L_{i}
$$

После факторизации Штейна слой $\varphi_{L}$ равен проходящей через $x$ компоненте множества (3.1), что и требовалось доказать.

Лемма 3.3. Пусть X - проективное гиперкэлерово многообразие, содержащее лагранжев подтор $L$. Тогда $L$-редукиия $\varphi_{L}: X \rightarrow \mathfrak{T}$ почти голоморбна.

ДокАЗАТЕльство. Пусть $\operatorname{dom}\left(\varphi_{L}\right)$ - область определения $\varphi_{L}$, a $Z:=X \backslash$ $\operatorname{dom}\left(\varphi_{L}\right)-$ множество, где $\varphi_{L}$ не определено. Надо показать, что общий слой отображения $\varphi_{L}$ не пересекает $Z$.

Рассуждая от противного, допустим, что для общей точки $x_{0} \in X \backslash X_{\Delta}$ проходящий через нее слой $F_{x_{0}}$ отображения $\varphi_{L}$ имеет нетривиальное пересечение с $Z$. Напомним, что множество $X_{\Delta}=\varepsilon\left(\mathfrak{U}_{\Delta}\right)$ заметается сингулярными деформациями тора $L$ согласно замечанию 2.4 , (i) и что отображение $\varphi_{L}$ голоморфно на $X \backslash X_{\Delta}$ согласно замечанию 3.1. Возьмем любую точку $z \in F_{x_{0}} \cap Z$ и рассмотрим график $X^{\prime} \subset X \times \mathfrak{T}$ отображения $\varphi_{L}$ с проекциями $p: X^{\prime} \rightarrow X$ и $\varphi_{L}^{\prime}: X^{\prime} \rightarrow \mathfrak{T}$. Как объясняется, например, в [10, п. 1.39], замкнутое подмножество $Z$ можно описать в виде

$$
Z=\left\{x \in X \mid \operatorname{dim} p^{-1}(x)>0\right\} .
$$

Поскольку $X$ нормально, а $p$ бирационально, $p$ имеет связные слои. Следовательно, многообразие $C^{\prime}:=\varphi_{L}^{\prime}\left(p^{-1}(z)\right)$ связно. Приведем еще некоторые свойства этого многообразия:

(i) $\operatorname{dim} C^{\prime}>0$, потому что $\operatorname{dim} p^{-1}(z)>0$, а $X^{\prime}-$ график $\varphi_{L}$;

(ii) $\varphi_{L}\left(x_{0}\right) \in C^{\prime}$ при $z \in F_{x_{0}}$;

(iii) $z$ содержится во всех слоях над точками из $C^{\prime}$.

Допустим, что справедлива диаграмма

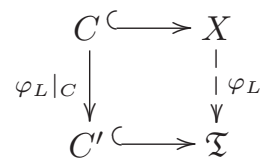

где $C$ связно, $x_{0} \in C$ и $\left.\varphi_{L}\right|_{C}-$ локальный изоморфизм в точке $x_{0}$. Покажем, что это допущение приводит к противоречию. Действительно, пусть $L_{1}, \ldots, L_{d}-$ $d=\operatorname{deg} \varepsilon$ различных торов из семейства $\left\{L_{t}\right\}_{t \in \mathfrak{B}}$, содержащих $x_{0}$. Поскольку $C$ связно и $C \not \subset F_{x_{0}}$ согласно свойству (i), из леммы 3.2 вытекает существование 
$k \in\{1, \ldots, d\}$ такого, что $C \not \subset L_{k}$. По лемме 2.3 малые деформации тора $L_{k}$ образуют слоение некоторой аналитической окрестности точки $x_{0}$. Значит, для каждой точки $y \in C \backslash\left\{x_{0}\right\}$, достаточно близкой к $x_{0}$, найдется малая деформация $L_{y}$ тора $L_{k}$ такая, что $y \in L_{y}$ и

$$
L_{y} \cap L_{k}=\varnothing .
$$

С другой стороны, по свойству (iii) и лемме 3.2 имеем

$$
z \in F_{y} \cap F_{x_{0}} \subset L_{y} \cap L_{k},
$$

что противоречит (3.3).

Остается найти нужное многообразие $C$. Заметим, что достаточно построить $C$ в евклидовой открытой окрестности точки $x_{0}$. Пользуясь общностью точки $x_{0}$ и теоремой о неявной функции, найдем малую окрестность $U \ni x_{0}$ такую, что сужение $\varphi_{L}: U \rightarrow V:=\varphi_{L}(U)$ является тривиальным голоморфным расслоением. В частности, множество $V \subset \mathfrak{T}$ открыто, и существует сечение $C \subset U$ для подмногообразия $C^{\prime} \cap V$. Остается лишь добиться связности множеств $C^{\prime} \cap V$ и $C$. Это можно сделать, уменьшая $V$ и $U$. Лемма доказана.

ОПредЕЛЕНИЕ 3.4. Проективное гиперкэлерово многообразие $X$, содержащее ларганжев подтор $L$, называется $L$-отделимым, если его $L$-редукция $\varphi_{L}: X \rightarrow \mathfrak{T}$ бирациональна.

3.2. Лагранжевы слоения на многообразиях, не являющихся $L$-отделимыми. Справедлива следующая

Теорема 3.5. Пусть $X$ - проективное гиперкэлерово многообразие $u$ $L \subset X$ - лагранжев подтор. Тогда $X$ допускает почти голоморфное слоение с сильным слоем $L$ в том и только в том случае, если $X$ не является L-отделимым.

Согласно последнему результату вопрос Бовилля можно переформулировать следующим образом.

Вопрос В. Существует ли такое проективное гиперкэлерово многообразие $X$ с лагранжевым подтором $L$, что $X$ является $L$-отделимым?

ДокаЗатЕЛЬСТво теОРемЫ 3.5 . Если $X$ не является $L$-отделимым, то $L$ редукция $\varphi_{L}: X \rightarrow \mathfrak{T}$ почти голоморфна (по лемме 3.3 ) и $0<\operatorname{dim} \mathfrak{T}<\operatorname{dim} X$. Тогда согласно [2, теорема 6.7] отображение $\varphi_{L}-$ почти голоморфное лагранжево слоение на $X$. Описание общего слоя $L$-редукции (лемма 3.2 ) показывает, что тор $L$ является сильным слоем $\varphi_{L}$.

Обратно, если $f: X \rightarrow B-$ почти голоморфное лагранжево слоение с сильным слоем $L$, то через общую точку проходит единственный лагранжев подтор в $\mathfrak{B}$ и $L$-редукция совпадает с рациональным отображением $\pi \circ \varepsilon^{-1}: X \rightarrow \mathfrak{B}$. В частности, $X$ не является $L$-отделимым. Теорема доказана.

\section{§4. Пересечение лагранжевых подторов}

Как и выше, пусть $X$ - проективное гиперкэлерово многообразие, содержащее лагранжев подтор $L$. В этом параграфе мы подробнее изучим окрестность $L$ в $X$, что даст ряд результатов о геометрии пересечений различных 
элементов семейства $\mathfrak{B}$ деформаций тора $L$. Будем всюду пользоваться обозначениями и результатами из п. 2.3 .

По лемме 2.3 семейство $\mathfrak{B}$ гладко в точке $[L]$ и найдется такая окрестность $V$ этой точки, что сужение $\left.\varepsilon\right|_{\mathfrak{U}_{V}}: \mathfrak{U}_{V} \rightarrow X$ вычисляющего отображения на прообраз $\mathfrak{U}_{V}:=\pi^{-1}(V)$ вкладывает $\mathfrak{U}_{V}$ в $X$. Таким образом, можно рассматривать $\mathfrak{U}_{V}$ как открытое подмножество в $X$. Пересечение множества $\mathfrak{U}_{V}$ с подмногообразием $M \subset X$ показано на рис. 1 .

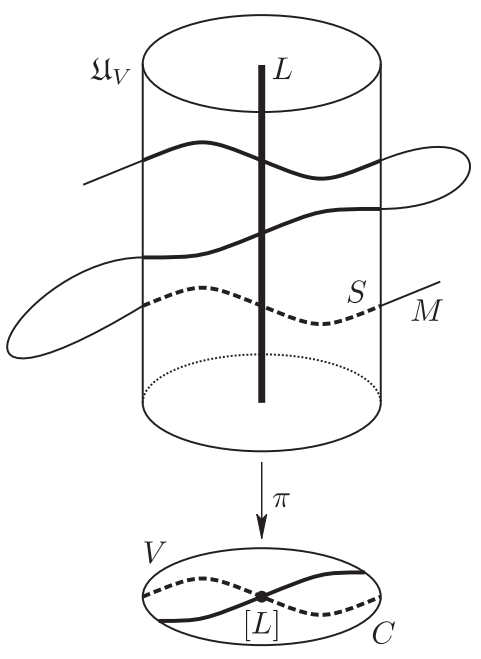

Рис. 1. Окрестность $\mathfrak{U}_{V}$ тора $L$ и ее проекция на $V \subset \mathfrak{B}$

ЛЕмма 4.1. Пусть $M \subseteq X$ - собственное гладкое подмногообразие и $L \subset X$ - гладкий тор, имеющий непустое пересечение с $M$. Тогда общая малая деформация тора $L$ имеет гладкое пересечение с $M$.

ДокАЗАТЕЛЬство. Пользуемся теми же обозначениями, что и выше. Поскольку множество $\mathfrak{U}_{V}$ открыто в $X$, пересечение $M \cap \mathfrak{U}_{V}$ гладко. Далее, отображение $\left.\pi\right|_{M \cap \mathfrak{U}_{V}}: M \cap \mathfrak{U}_{V} \rightarrow V$ собственное, поскольку $\pi$ собственное, а $M$ компактно. Следовательно, к $\left.\pi\right|_{M \cap \mathfrak{U}_{V}}$ применима теорема о гладкости в общем положении, откуда и вытекает требуемый результат.

ПредЛОЖЕНИЕ 4.2. Пусть $M \subseteq X$ - компактное подмногообразие $u$ $L \subseteq X$ - лагранжев подтор общего положения с $L \cap M \neq \varnothing$. Тогда расслоение $N_{L \cap M / M}$ тривиально. Если $M-$ комплексный тор, то $L \cap M-$ несвязное объединение торов.

ДокАзАтЕльство. В силу общности $L$ пересечение $L \cap M$ гладко по лемме 4.1. Более того, оба утверждения можно проверить, рассматривая каждый раз лишь одну связную компоненту множества $L \cap M$. Будем пользоваться обозначениями, введенными в начале параграфа, и рассмотрим любую связную компоненту $T$ множества $L \cap M$. Если окрестность $V$ достаточно мала, то включение $L \cap M \hookrightarrow \mathfrak{U}_{V} \cap M$ индуцирует взаимно однозначное соответствие связных компонент. Пусть $S$ - связная компонента множества $\mathfrak{U}_{V} \cap M$, соответствующая $T$. В силу общности $L$ можно считать отображение $\left.\pi\right|_{S}$ гладким, 
так что множество $C:=\pi(S) \subset V$ является гладким многообразием размерности $n-\operatorname{dim} T$ вблизи $[L]$. При этом $C$ параметризует те малые деформации тора $L$, которые задают плоскую деформацию $T$ внутри $M$. Таким образом, получаем, что семейству $S \rightarrow C$ соответствует классифицирующее отображение $\chi: C \rightarrow \mathscr{D}(M)$ из $C$ в пространство Дуади многообразия $M$.

На уровне касательных пространств имеем $T_{C}([L]) \subset T_{\mathfrak{B}}([L])=H^{0}\left(L, N_{L / X}\right)$, где последнее равенство происходит из морфизма Гильберта-Чжоу (cp. [2, лемма 3.1]). Морфизм $\chi$ индуцирует отображение $\chi_{*}: T_{C}([L]) \rightarrow H^{0}\left(T, N_{T / M}\right)$, но малые деформации $T$ внутри $M$, индуцированные деформациями тора $L$, не пересекаются с $T$ по лемме 2.3 , (ii). Поэтому отображение $\chi_{*}$ инъективно и образ $T_{C}([L])$ состоит из нигде не обращающихся в нуль сечений. По соображениям размерности эти сечения порождают всё нормальное расслоение подмногообразия $T$ в $M$. Следовательно, расслоение $N_{T / M}$ тривиально, что и требовалось показать.

Если $M$ также является тором, то аналогично получаем тривиальность $\left.T_{M}\right|_{T}$. Поэтому согласно последовательности нормального расслоения

$$
\left.0 \longrightarrow T_{T} \longrightarrow T_{M}\right|_{T} \longrightarrow N_{T / M} \longrightarrow 0
$$

касательное расслоение $T_{T}$ тривиально и, тем самым, $T$ - комплексный тор. Предложение доказано.

Пользуясь предложением 4.2, уточним наблюдение из замечания 2.4, (ii).

Лемма 4.3. Пусть $X$ - четырехмерное гиперкэлерово многообразие, $L u$ $M$ - лагранжевы гладко пересекающиеся торы, и пусть $I=L \cap M$. Тогда I конечное несвязное объединение эллиптических кривых.

ДокАЗАтельство. Осталось лишь исключить возможность существования нульмерных связных компонент у множества $I$. Согласно общей теории лагранжевых пересечений (см., например, [11, введение]) имеем

$$
[L] \cdot[M]=\chi(I) .
$$

Однако отсюда уже вытекает требуемое, так как в силу предложения 4.2 всякая компонента положительной размерности множества $I$ является гладкой эллиптической кривой и ее вклад в эйлерову характеристику $\chi(I)$ равен нулю. Лемма доказана.

СлЕДСТвИЕ 4.4. Пусть $X$ - четырехмерное проективное гиперкэлерово многообразие, $L$ - лагранжев подтор. Если $X$ является L-отделимым, то вычисляющее отображсние $\varepsilon$ из диаграммы (2.2) имеет степень не меньше mpex.

ДокАЗАТЕЛЬСтво. В силу $L$-отделимости $X$ из теоремы 3.5 и леммы 2.5 вытекает, что отображение $\varepsilon$ не бирационально. Остается исключить случай $\operatorname{deg} \varepsilon=2$. По лемме $3.2 L$-отделимость означает, что в общей точке $x \in X$ связная компонента множества $\bigcap_{[M] \in \mathfrak{B}, x \in M} M$ равна $x$. Если $\operatorname{deg} \varepsilon=2$, то существуют только два тора в $\mathfrak{B}$, содержащих $x$, скажем $M_{1}$ и $M_{2}$. Поскольку точка $x$ общего положения, пересечение $M_{1} \cap M_{2}$ гладко по лемме 4.1. Тогда лемма 4.3 противоречит тому, что связная компонента множества $M_{1} \cap M_{2}$, содержащая $x$, равна $\{x\}$. 


\section{§ 5. Четырехмерные гиперкэлеровы многообразия}

C помощью результатов из $\S 4$ мы докажем теперь наш основной результат, дающий положительный ответ (в самом сильном возможном смысле) на вопрос Бовилля.

Теорема 5.1. Пусть $X$ - четырехмерное гиперкэлерово многообразие, содержащее лагранжев тор L. Тогда X допускает голоморфное лагранжево слоение со слоем L.

ЗАмечАниЕ 5.2. Мы благодарны Е. Америк, сообщившей нам следующее наблюдение из линейной алгебры, которое позволяет исключить $L$-отделимость многообразий $X \supset L$ в размерности четыре. Это сильно упростило наше первоначальное теоретико-деформационное доказательство.

Лемма 5.3. Пусть $V$ - четырехмерное симплектическое векторное пространство с симплектической формой $\sigma, a W_{1}, W_{2}, W_{3} \subset V-$ три лагранжевых подпространства, причем $\operatorname{dim} W_{i} \cap W_{j}=1$ для всех $i \neq j$. Тогда $W_{1} \cap W_{2} \cap W_{3} \neq\{0\}$.

ДоказАтельство. Допустим от противного, что $W_{1} \cap W_{2} \cap W_{3}=\{0\}$, и рассмотрим линейную оболочку $\left\langle W_{1}, W_{2}\right\rangle$. Она трехмерна, так как по условию $\operatorname{dim} W_{1} \cap W_{2}=1$. Покажем, что

$$
W_{3} \subset\left\langle W_{1}, W_{2}\right\rangle .
$$

Действительно, иначе было бы $\operatorname{dim} W_{3} \cap\left\langle W_{1}, W_{2}\right\rangle=1$, откуда все пересечения $W_{3} \cap\left\langle W_{1}, W_{2}\right\rangle=W_{3} \cap W_{1}=W_{3} \cap W_{2}$ одномерны вопреки нашему предположению о том, что $W_{1} \cap W_{2} \cap W_{3}=\{0\}$.

Теперь, также пользуясь равенством $W_{1} \cap W_{2} \cap W_{3}=\{0\}$, запишем $\left\langle W_{1}, W_{2}\right\rangle=$ $W_{3} \oplus\left(W_{1} \cap W_{2}\right)$. Поскольку $V$ симплектично, а $W_{3}$ лагранжево, найдутся $v \in W_{1} \cap W_{2}$ и $w \in W_{3}$ такие, что $\sigma(v, w) \neq 0$. Согласно включению (5.1) можно записать $w=w_{1}+w_{2}$, где $w_{i} \in W_{i}$. Тогда

$$
0 \neq \sigma(v, w)=\sigma\left(v, w_{1}\right)+\sigma\left(v, w_{2}\right)=0+0=0,
$$

поскольку $W_{1}$ и $W_{2}$ лагранжевы. Противоречие. Лемма доказана.

ЗАмЕчание 5.4. Можно также доказать лемму 5.3 следующим красивым геометрическим рассуждением, которое рассказал нам Л. Манивель. Грассманиан лагранжевых подпространств в $V \cong \mathbb{C}^{4}$ биголоморфен (гладкому) пересечению квадрики Плюккера $\widetilde{Q} \subset \mathbb{P}\left(\bigwedge^{2} V\right)$ и векторного подпространства, заданного обращением в нуль симплектической формы $\sigma: \bigwedge^{2} V \rightarrow \mathbb{C}$. Следовательно, он является гладкой квадрикой $Q \subset \mathbb{P}^{4}$. Условие $\operatorname{dim} W_{i} \cap W_{j}=1$ означает, что прямая в $\mathbb{P}^{4}$, соединяющая точки $\left[W_{i}\right],\left[W_{j}\right] \in Q$, содержится в $Q$. Если $W_{1} \cap W_{2} \cap W_{3}=\{0\}$, то на $\left[W_{1}\right],\left[W_{2}\right],\left[W_{3}\right]$ натягивается некоторая плоскость $P$. Однако многообразие $P \cap Q$ имеет степень 2 и, следовательно, не может содержать объединение трех прямых.

ПреДЛОЖЕНИЕ 5.5. Пусть $X$ - проективное четырехмерное гиперкэлерово многообразие, содержащее лагранжсе тор $L$. Тогда $X$ не является $L$-отделимым.

ДокАЗАтельство. Допустим от противного, что $X L$-отделимо. Для общей точки $x \in X$ по лемме 3.2 и следствию 4.4 найдутся натуральное число $d \geqslant 3$ и $d$ гладких лагранжевых подторов, локально высекающих $x$. В силу 
общности $x$ леммы 4.1, 4.3 дают нам два таких тора, скажем $L_{1}$ и $L_{2}$, которые пересекаются по эллиптической кривой $E$ в точке $x$. Всякий другой тор из $\mathfrak{B}$, проходящий через $x$, либо содержит $E$, либо высекает нульмерную подсхему.

В силу $L$-отделимости существует лагранжев тор $L_{3}$, содержащий точку $x$, но не содержащий $E$ и такой, что схема-пересечение $L_{1} \cap L_{2} \cap L_{3}$ нульмерна в точке $x$. Опять пользуясь общностью $x$, мы можем считать пересечения $L_{1} \cap L_{3}, L_{2} \cap L_{3}$ и $L_{1} \cap L_{2} \cap L_{3}$ гладкими в точке $x$. Следовательно, три лагранжевых подпространства $W_{i}:=T_{L_{i}, x} \subset T_{X, x}$ удовлетворяют условиям леммы 5.3. Отсюда вытекает, что $W_{1} \cap W_{2} \cap W_{3} \neq\{0\}$ вопреки нашему выбору торов $L_{1}$, $L_{2}, L_{3}$. Следовательно, $X$ не может быть $L$-отделимым.

ДокАЗАТЕЛЬСтво тЕоремы 5.1. Если многообразие $X$ не проективно, то нужное утверждение вытекает из [2, теорема 4.1]. Поэтому будем считать $X$ проективным. По предложению 5.5 многообразие $X$ не является $L$-отделимым и, следовательно, допускает почти голоморфное лагранжево слоение $f: X \rightarrow B$ по теореме 3.5. Остается показать, что из существования почти голоморфного лагранжева слоения вытекает существование голоморфного. Это доказано в следующей лемме.

Лемма 5.6. Пусть $f: X \rightarrow B$ - почти голоморфное лагранжсево слоение на четырехмерном проективном гиперкэлеровом многообразии. Тогда существует бирачиональная модификация $\psi: B \rightarrow B^{\prime}$ такая, что $\psi \circ f: X \rightarrow B^{\prime}-$ голоморфное лагранжево слоение.

Доказательство леммы 5.6 опирается на явное описание бирациональной геометрии четырехмерных гиперкэлеровых многообразий. Для формулировки этого описания напомним понятие флопа Myкаu. Пусть четырехмерное гиперкэлерово многообразие $X$ содержит гладкое алгебраическое подмногообразие $P \cong \mathbb{P}^{2}$. Если раздуть $P$, то исключительный дивизор будет изоморфен проективному расслоению $\mathbb{P}\left(\Omega_{\mathbb{P}^{2}}^{1}\right)$, и хорошо известно, что существует его сдутие в другом направлении, дающее новое гиперкэлерово многообразие $X^{\prime}$. Полученное бирациональное преобразование $X \rightarrow X^{\prime}$ называется флопом Мукаи относительно $P$.

ДокАЗАТЕЛЬСтво ЛЕммы 5.6. Согласно [2, п. 6.2] существуют голоморфная модель для $f$, т. е. лагранжево слоение $f^{\prime}: X^{\prime} \rightarrow B^{\prime}$ на (возможно, другом) гиперкэлеровом многообразии $X^{\prime}$, и диаграмма

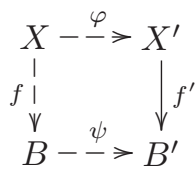

с бирациональными горизонтальными стрелками такими, что $\varphi$ является изоморфизмом вблизи общего слоя отображения $f$.

Покажем, что композиция $f^{\prime} \circ \varphi=\psi \circ f$ голоморфна и, следовательно, является лагранжевым слоением на $X$. Для этого заметим сначала, что согласно [12, теорема 1.2] отображение $\varphi$ разлагается в конечную композицию флопов Мукаи. Поэтому можно считать по индукции, что $\varphi^{-1}-$ одновременный флоп Мукаи относительно несвязного объединения вложенных проективных плоскостей $\mathbb{P}^{2} \cong P_{i} \subset X^{\prime}$. 
В силу голоморфности $\varphi^{-1}$ вблизи общего слоя $f^{\prime}$ ни одна из плоскостей $P_{i}$ не может пересекать общий слой. Поэтому $f^{\prime}\left(P_{i}\right)$ является собственным подмножеством $B^{\prime}$ и, следовательно, имеет размерность не выше 1 . Поскольку непостоянных отображений из $\mathbb{P}^{2}$ в кривую не существует, $f^{\prime}\left(P_{i}\right)$ - одна точка. Иными словами, множество точек неопределенности отображения $\varphi^{-1}$ содержится в слоях $f^{\prime}$, так что композиция $f^{\prime} \circ \varphi$ остается голоморфной.

\section{Список литературы}

1. A. Beauville, "Holomorphic symplectic geometry: a problem list", Complex and differential geometry, Springer Proc. Math., 8, Springer-Verlag, Heidelberg, 2011, 49-63.

2. D. Greb, Ch. Lehn, S. Rollenske, Lagrangian fibrations on hyperkahler manifolds - On a question of Beauville, arXiv: abs/1105.3410.

3. E. Amerik, F. Campana, "Fibrations méromorphes sur certaines variétés à fibré canonique trivial", Pure Appl. Math. Q., 4:2, part 1 (2008), 509-545.

4. E. Amerik, A remark on a question of Beauville about lagrangian fibrations, arXiv: abs/1110.2852.

5. Jun-Muk Hwang, R. Weiss, Webs of Lagrangian tori in projective symplectic manifolds, arXiv: abs/1201.2369.

6. D. Matsushita, "On fibre space structures of a projective irreducible symplectic manifold", Topology, 38:1 (1999), 79-83.

7. D. Matsushita, "Equidimensionality of Lagrangian fibrations on holomorphic symplectic manifolds", Math. Res. Lett., 7:4 (2000), 389-391.

8. D. Matsushita, "Addendum: "On fibre space structures of a projective irreducible symplectic manifold"", Topology, 40:2 (2001), 431-432.

9. D. Matsushita, "Holomorphic symplectic manifolds and Lagrangian fibrations", Acta Appl. Math., 75:1-3 (2003), 117-123.

10. O. Debarre, Higher-dimensional algebraic geometry, Universitext, Springer-Verlag, New York, 2001.

11. K. Behrend, B. Fantechi, "Gerstenhaber and Batalin-Vilkovisky structures on Lagrangian intersections", Algebra, arithmetic, and geometry: in honor of Yu. I. Manin, v. I, Progr. Math., 269, Birkhäuser, Boston, MA, 2009, 1-47.

12. J. Wierzba, J. A. Wiśniewski, "Small contractions of symplectic 4-folds", Duke Math. J., 120:1 (2003), 65-95.

ДАНИЕЛЬ ГРЕБ

(Daniel Greb)

A. Ludwigs University of Freiburg, Germany

E-mail: daniel.greb@math.uni-freiburg.de

ХрИСТИАН ЛЕН

(Christian Lehn)

Institut Fourier, France

E-mail: christian.lehn@ujf-grenoble.fr

Зёнке РолЛЕнске

(Sonke Rollenske)

Department of Mathematics, Bielefeld University,

Germany

E-mail: rollenske@math.uni-bielefeld.de

Перевод с английского А. В. Домрина
Поступило в редакцию 02.03.2012 